\title{
Electronic speckle pattern interferometry for fracture expansion in nuclear graphite based on PDE image processing methods
}

Chen Tang, Junjiang Zhang, Chen Sun, Yonggang Su, Kai Leung Su

Chen Tang, Junjiang Zhang, Chen Sun, Yonggang Su, Kai Leung Su, "Electronic speckle pattern interferometry for fracture expansion in nuclear graphite based on PDE image processing methods," Proc. SPIE 9525, Optical Measurement Systems for Industrial Inspection IX, 95252Y (22 June 2015); doi: $10.1117 / 12.2184552$

SPIE. Event: SPIE Optical Metrology, 2015, Munich, Germany 


\title{
Electronic speckle pattern interferometry for fracture expansion in nuclear graphite based on PDE image processing methods
}

\author{
Chen Tang*a, Junjiang Zhang ${ }^{\mathrm{a}}$, Chen Sun ${ }^{\mathrm{a}}$, Yonggang Su ${ }^{\mathrm{a}}$, SU Kai Leung ${ }^{\mathrm{b}}$ \\ ${ }^{a}$ Department of Applied Physics, University of Tianjin, Tianjin, 300072, P.R.China \\ ${ }^{\mathrm{b}}$ Department of Civil Engineering, University of Hong Kong, Hong Kong, 999077, P.R.China \\ *Corresponding author: tangchen@tju.edu.cn
}

\begin{abstract}
Nuclear graphite has been widely used as moderating and reflecting materials. However, due to severe neutron irradiation under high temperature, nuclear graphite is prone to deteriorate, resulting in massive microscopic flaws and even cracks under large stress in the later period of its service life. It is indispensable, therefore, to understand the fracture behavior of nuclear graphite to provide reference to structural integrity and safety analysis of nuclear graphite members in reactors. In this paper, we investigated the fracture expansion in nuclear graphite based on PDE image processing methods. We used the secondorder oriented partial differential equations filtering model (SOOPDE) to denoise speckle noise, then used the oriented gradient vector fields for to obtain skeletons. The full-field displacement of fractured nuclear graphite and the location of the crack tip were lastly measured under various loading conditions.
\end{abstract}

Keywords: Electronic speckle pattern interferometry, the oriented partial differential equations image processing, filtering speckle noise, fractured nuclear graphite, the location of the crack tip.

\section{INTRODUCTION}

At present, energy shortage has become the most severe challenge. Developing nuclear power generation provides a viable solution to the problem and is greatly supported by various governments. The task of nuclear engineering is to safely and effectively utilize the nuclear fission to acquire tremendous energy. The utilization ratio of neutrons, which controls the effectiveness of the nuclear fission, can be enhanced by using moderating and reflecting materials in the reactor core. Nuclear graphite which processes large neutron scattering cross-section, small neutron absorption cross-section, low mass number, high atomic density per unit volume and excellent mechanical performance under high temperature has been widely used in reactor core as moderating and reflecting materials. However, due to the severe neutron irradiation under high temperature in and around reactor core, nuclear graphite is prone to deteriorate and results in massive microscopic flaws and even fracture under large stress in the later period of its service life. Thus, the safety of nuclear power station is threatened. It is indispensable, therefore, to understand the fracture behavior of nuclear graphite to provide reference to the structural integrity and safety analysis of nuclear graphite members in reactors.

Determining the full-field displacement, crack tip position and crack mouth opening displacement of fractured nuclear graphite is the key issue of fracture mechanics of nuclear graphite, which is particularly important to apprehend the fracture mechanism of nuclear graphite and establish the principles of fracture stability analysis and safety evaluation of nuclear graphite members. Electronic speckle pattern interferometry (ESPI) as a well-known technique for the measurement of deformation fields of the object surface, has been extensively investigated and widely used in numerous fields for its simple optic devices and its resistance ability to environment noise. Accurate extraction of phase values is very important because it is related with a physical quantity. The fringe skeleton method may be the most straightforward approach ${ }^{[1]}$. This method offers the advantage in that there is no need to perform phase stepping. To perform fringe skeleton analysis automatically, the image is first processed to remove noise. Then skeletons are extracted and fringe numbers are assigned starting from the point where no external load is or where absolute deformation is known. Lastly, an interpolation can be performed to obtain phase values for the whole field. Obviously, skeletonization of fringes has played an important role in the fringe skeleton 
method. For obtaining desired skeletons, the removals of massive speckle noise in ESPI fringe patterns are usually needed. The partial differential equation (PDE) methods developed recently have been demonstrated to be a powerful tool for optical interferometry fringe processing. In this paper, we investigated the fracture expansion in nuclear graphite based on PDE image processing methods. We used the second-order oriented partial differential equations filtering model (SOOPDE) to denoise speckle noise, then used the oriented gradient vector fields for to obtain skeletons. The full-field displacement of fractured nuclear graphite and the location of the crack tip were lastly measured under various loading conditions.

\section{DESCRIPTION OF THE METHOD}

In this section, we describe the method for extracting phase values based on the fringe skeleton method. Let us briefly describe the overall procedure. Firstly, utilize the oriented bilateral filtering method and SOOPDE to ESPI fringe patterns and obtain the filtered images. Secondly, extract skeletons by the gradient vector fields method. Thirdly, assign order for skeleton map. Fourthly, obtain corresponding unwrapped phase values by $\mathrm{C}$ spline interpolation.

\subsection{Denoise speckle noise}

For various loading conditions, ESPI fringe patterns have various densities. For the fringe patterns with ordinary density, we use the oriented bilateral filtering model, and for the fringe patterns with high density, we use SOOPDE to remove speckle noise.

Bilateral filtering ${ }^{[3,4]}$ is a popular edge-preserving filter where the weight of each pixel is computed using a Gaussian in the spatial domain multiplied by an influence function in the intensity domain that decreases the weight of pixels with large intensity differences. Owe to its simplicity and easy implementation, it has been widely used in image processing and analysis. Bilateral filtering is a non-linear filter where the output is a weighted average of the input.The weighting for each pixel $q$ is determined by the spatial distance from the center pixel $p$, as well as its relative difference in intensity. Let $I_{p}$ be the intensity at pixel $p$, and $B F[I]_{p}$ be the filtered value that is defined by

$$
B F[I]_{p}=W_{p}^{-1} \sum_{q \in S} G_{\sigma_{d}}(\|p-q\|) G_{\sigma_{r}}\left(I_{p}-I_{q}\right) I_{q},
$$

where $G_{\sigma_{d}}$ and $G_{\sigma_{r}}$ are two Gaussian filters at a localized pixel neighborhood, $p-q$ and $I_{p}-I_{q}$ are respectively the Euclidean distance and the Gray difference for the two pixels of the image, $\sigma_{d}$ and $\sigma_{r}$ are standard deviation based on the Gaussian function. Here we establish a mean mask along the fringe orientation. Apply the idea of the direction mask to the traditional bilateral filtering method, we can obtain the oriented bilateral filtering method.

In [2], Tang et.al proposed the second-order oriented PDE models (SOOPDE). That is

$$
u_{t}=u_{x x} \cos ^{2} \theta+u_{y y} \sin ^{2} \theta+2 u_{x y} \sin \theta \cos \theta,
$$

where $u(x, y, t)$ is the evolving image, $\theta$ is the angle between the fringe orientation with $x$ coordinate, $u_{x x}$ and $u_{y y}$ are the second order partial derivatives of $u(x, y, t)$ with respect to coordinates $x$ and $y, u_{x y}$ is the second order mixed partial derivative.

Fig. 1(a, b, c) shows the three experimentally obtained ESPI speckle fringe patterns with very poor quality. Fig. 1(d) is the filtered image by the the oriented bilateral filtering model. And Figs. 1(e) and 1(f) are respective the filtered images by the SOOPDE. 


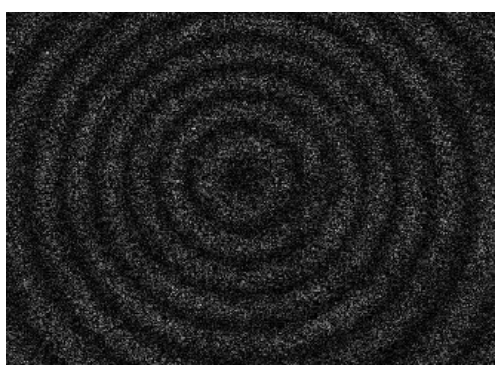

(a)

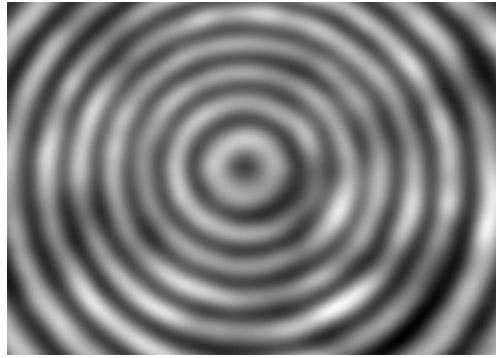

(d)

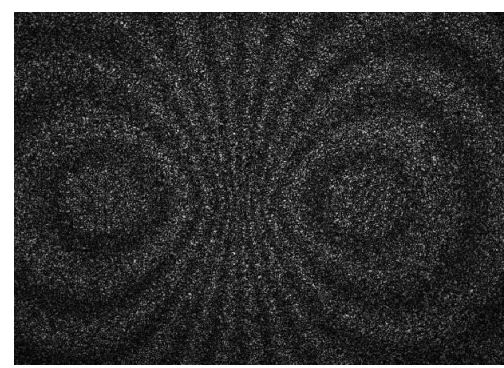

(b)

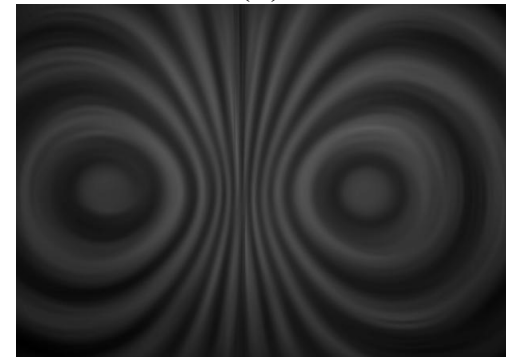

(e)

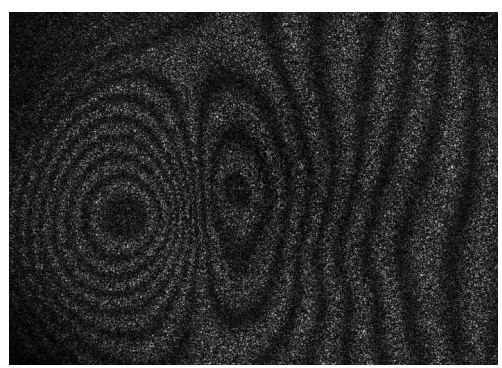

(c)

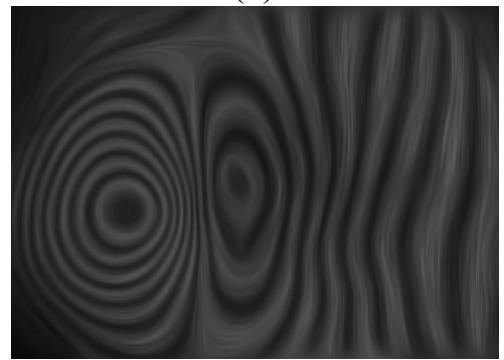

(f)

Figure 1. The three experimentally obtained ESPI speckle fringe patterns and their filtered images.

\subsection{Extract skeleton}

There are many techniques to extract the fringe skeletons. The fringe extreme tracking method and threshold binary-fringe thinning method are the two well-known and widely used methods used to extract the fringe skeletons. The fringe extreme tracking method is to identify peaks by finding the maximum or minimum individual pixel value in a neighborhood. The threshold binary-fringe and thinning method uses a predetermined threshold to make a binary-fringe pattern. Then, the binary fringes are thinned to single lines. However, for the ESPI fringe patterns with low quality, the skeletons, obtained by the two methods are very poor.

Recently, the skeletonization methods of gray scale image based on PDEs have been actively studied. The most main advantage of this method is its simplicity. It works directly on gray-scale images without the segmentation stage. The basic ideal of the methods is that gradient vector fields of a given image are calculated (or called diffused) by a set of linear or nonlinear governing PDEs. Then these gradient vector fields are used for the extraction of skeletons based on the topological analysis of $\mathrm{GVFs}^{[5]}$. The gradient vector field was firstly defined as the vector field by $\mathrm{Xu}$ and Prince for active contour models,

$$
\mathbf{V}(x, y)=[u(x, y), v(x, y)]=\left[\frac{\partial I}{\partial x}, \frac{\partial I}{\partial y}\right] .
$$

In Ref.[6], we proposed coupled nonlinear governing PDEs for ESPI fringe patterns with usual density.

$$
\begin{aligned}
& \frac{\partial u(x, y, t)}{\partial t}=\alpha g\left(\left|\nabla u^{\prime}\right|\right)|\nabla u| \operatorname{div}\left(\frac{\nabla u}{|\nabla u|}\right)+\alpha \nabla\left(g\left(\left|\nabla u^{\prime}\right|\right)\right) \nabla u-\beta\left(u-I_{x}\right)|\nabla u|+\gamma \operatorname{div}\left(g\left(\left|\nabla u^{\prime}\right|\right) \nabla u\right), \\
& \frac{\partial u(x, y, t)}{\partial t}=\alpha g\left(\left|\nabla u^{\prime}\right|\right)|\nabla u| \operatorname{div}\left(\frac{\nabla u}{|\nabla u|}\right)+\alpha \nabla\left(g\left(\left|\nabla u^{\prime}\right|\right)\right) \nabla u-\beta\left(u-I_{x}\right)|\nabla u|+\gamma \operatorname{div}\left(g\left(\left|\nabla u^{\prime}\right|\right) \nabla u\right), \\
& \frac{\partial u(x, y, t)}{\partial t}=\alpha g\left(\left|\nabla u^{\prime}\right|\right)|\nabla u| \operatorname{div}\left(\frac{\nabla u}{|\nabla u|}\right)+\alpha \nabla\left(g\left(\left|\nabla u^{\prime}\right|\right)\right) \nabla u-\beta\left(u-I_{x}\right)|\nabla u|+\gamma \operatorname{div}\left(g\left(\left|\nabla u^{\prime}\right|\right) \nabla u\right),
\end{aligned}
$$




$$
\frac{\partial u(x, y, t)}{\partial t}=\alpha g\left(\left|\nabla u^{\prime}\right|\right)|\nabla u| \operatorname{div}\left(\frac{\nabla u}{|\nabla u|}\right)+\alpha \nabla\left(g\left(\left|\nabla u^{\prime}\right|\right)\right) \nabla u-\beta\left(u-I_{x}\right)|\nabla u|+\gamma \operatorname{div}\left(g\left(\left|\nabla u^{\prime}\right|\right) \nabla u\right) .
$$

Further, in Ref.[7] we proposed the oriented couple governing PDEs for ESPI fringe patterns with high density,

$$
\begin{gathered}
\frac{\partial u}{\partial t}=\alpha g\left(\left|\nabla u^{\prime}\right|\right)\left(u_{x x} \cos ^{2} \theta+u_{y y} \sin ^{2} \theta+2 u_{x y} \sin \theta \cos \theta\right)-\beta\left(u-I_{x}\right)|\nabla u|, \\
\frac{\partial v}{\partial t}=\alpha g\left(\left|\nabla v^{\prime}\right|\right)\left(v_{x x} \cos ^{2} \theta+v_{y y} \sin ^{2} \theta+2 v_{x y} \sin \theta \cos \theta\right)-\beta\left(v-I_{y}\right)|\nabla v|, \\
\frac{\partial u^{\prime}}{\partial t}=a(t)\left(u_{x x}^{\prime} \cos ^{2} \theta+u_{y y}^{\prime} \sin ^{2} \theta+2 u_{x y}^{\prime} \sin \theta \cos \theta\right)-b\left(u^{\prime}-u\right), \\
\frac{\partial v^{\prime}}{\partial t}=a(t)\left(v_{x x}^{\prime} \cos ^{2} \theta+v_{y y}^{\prime} \sin ^{2} \theta+2 v_{x y}^{\prime} \sin \theta \cos \theta\right)-b\left(v^{\prime}-v\right),
\end{gathered}
$$

where $u(x, y, t)$ and $v(x, y, t)$ are two components of the gradient vector field. $\alpha, \beta, \gamma$ and $b$ are the constant parameters, $a(t)$ is a parameter which changes with time. The function $g\left(\left|\nabla u^{\prime}\right|\right)$ and $g\left(\left|\nabla v^{\prime}\right|\right)$ are nonincreasing function of the gradient $\left|\nabla u^{\prime}\right|$ and $\left|\nabla v^{\prime}\right|$, respectively. After calculating the gradient vector field, the GVF is normalized. The normalized gradient vector field (NGVF) can describe the geometric structures of boundary and the skeleton of an object very well. Given the threshold, say $\bar{T}$, the skeleton image of white fringes $f_{\mathrm{WFS}}(x, y)$, the skeleton image of black fringes $f_{\mathrm{BFS}}(x, y)$ and the skeleton image of all fringes $f_{\mathrm{FS}}(x, y)$ are obtained through the following rules, respectively $\mathrm{y}^{[7]}$,

$$
\begin{aligned}
& f_{\mathrm{WFS}}(x, y)=\left\{\begin{array}{cc}
1, \text { skeleton pixel } & \text { if } \mathrm{S}(x, y)>\bar{T} \text { and } J M(x, y)<0, \\
0, \text { non-skeleton pixel } & \text { otherwise }
\end{array}\right.
\end{aligned}
$$

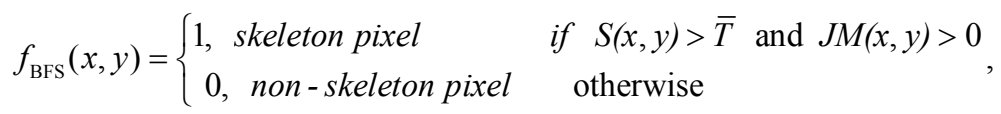

$$
\begin{aligned}
& f_{\mathrm{FS}}(x, y)=\left\{\begin{array}{lc}
1, \text { skeleton pixel } & \text { if } S(x, y)>\bar{T} \\
0, \text { non }- \text { skeleton pixel } & \text { otherwise }
\end{array},\right.
\end{aligned}
$$

where $\bar{T}$ is a given threshold,

$$
\begin{gathered}
S(x, y)=\frac{\left(\frac{\partial u_{N}(x, y)}{\partial x}\right)^{2}+\left(\frac{\partial v_{N}(x, y)}{\partial y}\right)^{2}}{\max \left(\left(\frac{\partial u_{N}(x, y)}{\partial x}\right)^{2}+\left(\frac{\partial v_{N}(x, y)}{\partial y}\right)^{2}\right)}, \\
J M(x, y)=\left(\frac{\partial u_{N}(x, y)}{\partial x}+\frac{\partial v_{N}(x, y)}{\partial y}\right)- \\
\frac{\operatorname{real} \sqrt{\left(\frac{\partial u_{N}(x, y)}{\partial x}\right)^{2}-2\left(\frac{\partial u_{N}(x, y)}{\partial x}\right)\left(\frac{\partial v_{N}(x, y)}{\partial y}\right)+\left(\frac{\partial v_{N}(x, y)}{\partial y}\right)^{2}+4\left(\frac{\partial u_{N}(x, y)}{\partial y}\right)\left(\frac{\partial v_{N}(x, y)}{\partial x}\right)}}{\lambda},
\end{gathered}
$$


where $\lambda$ is a constant. In this paper, all orientation angles are calculated by the Fourier transform method.

Fig. 2(a) is an experimentally obtained original ESPI fringe image, and Fig. 2(b) is its filtered image using a $3 \times 3$ window mean filtering for only one time. The black fringe and white fringe skeletons of Fig. 2(b) are shown in Fig. 2(c) and Fig. 2(d).

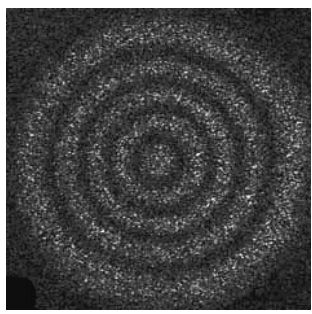

(a)

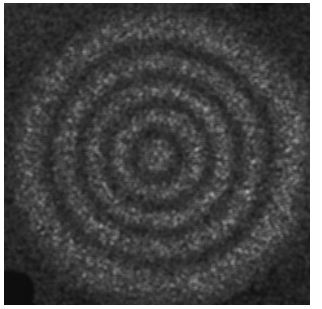

(b)

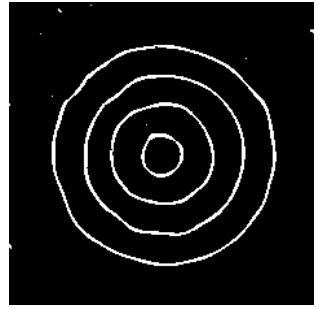

(c)

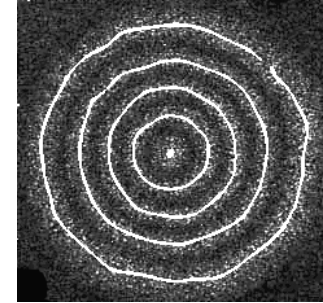

(d)

Figure 2. An experimentally obtained ESPI fringe pattern and its skeletons, (a) Initial image; (b) The filtered image of Fig.2(a) using a mean filtering for only one time; (c) The skeletons of black fringes of Fig.2(b) by PDEs (4-7); (d) The skeletons of white fringes of Fig.2(b) by PDEs (4-7).

Fig. 3(a) is an experimentally obtained original interferometric fringe image using Michelson Interferometer. After normalizing and mean filtering with $3 \times 3$ window for 5 times, its binary image by the dot thresholding method ${ }^{[8]}$ is shown in Fig. 3(b). The white and black fringe skeletons of Fig. 3(b) by Hilditch thinning are given in Fig. 3(c) and Fig. 3(d), respectively. Fig. 3(e) and Fig. 3(f) show the white and black fringe skeletons by the extreme tracking method, respectively. The results by our governing PDEs (8-11) are given in Fig. 3(g) and Fig. 3(h), respectively.

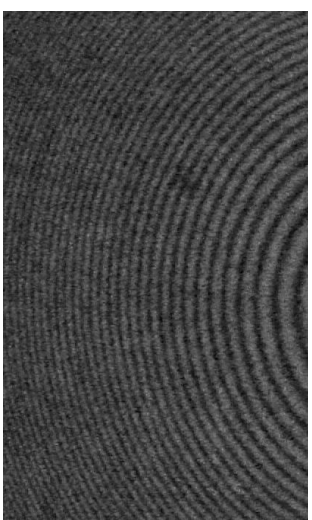

(a)

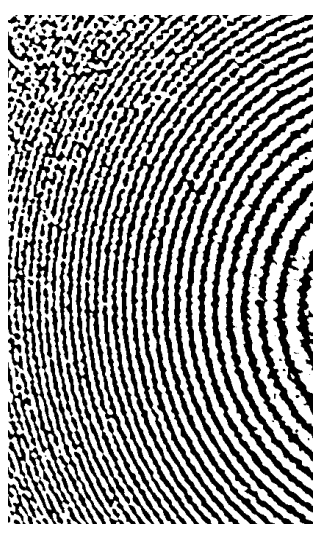

(b)

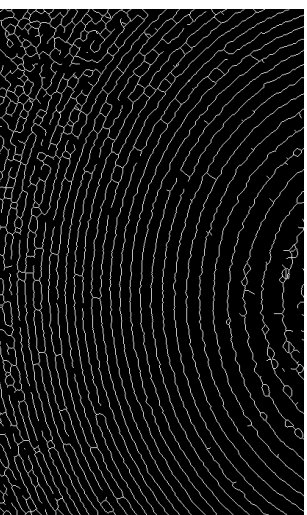

(c)

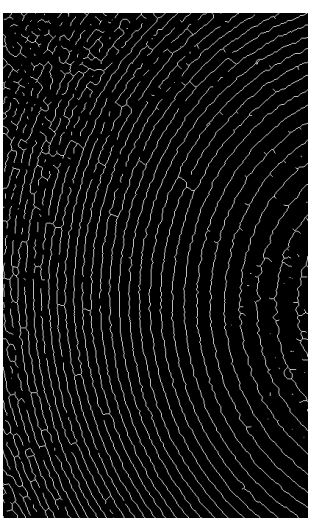

(d) 


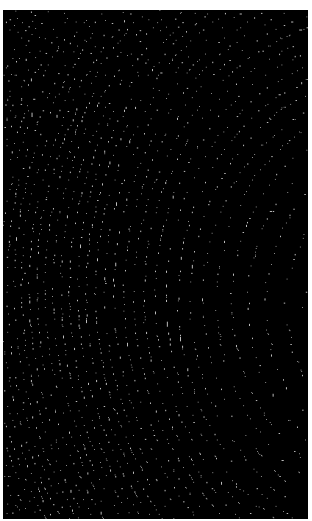

(e)

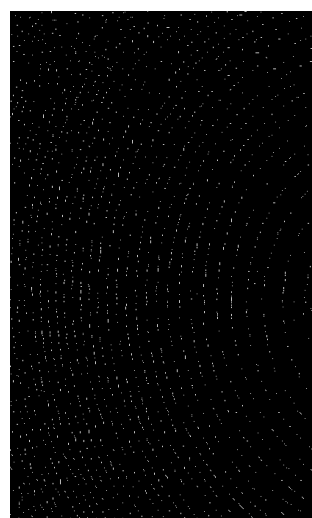

(f)

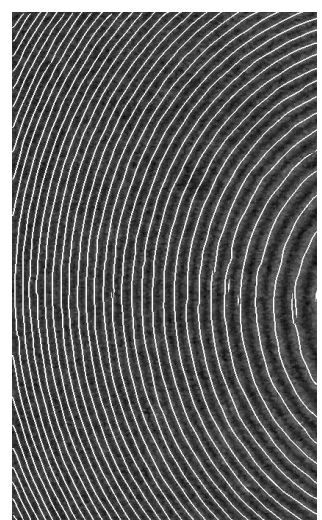

(g)

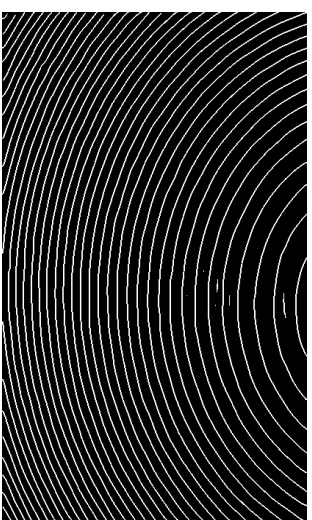

(h)

Figure 3. An original interferometric fringe image and its skeletons by various methods. (a)Initial image; The binary image of Fig. 2(a); (c) The skeletons of white fringes of Fig. 3(b) by Hilditch thinning method; (d) The skeletons of black fringes of Fig. 3(b) by Hilditch thinning method; (e) The skeletons of white fringes of filtered Fig. 3(a) by the fringe extreme tracking method; (f) The skeletons of black fringes of filtered Fig.3 (a) by the fringe extreme tracking method; (g) The skeletons of white fringes of Fig. 3(a) by PDEs (8-11); (h) The skeletons of black fringes of Fig. 3(a) by PDEs (8-11).

\section{EXPERIMENTS AND DICUSSION}

Figure 4 shows the ESPI fringe images of fractured nuclear graphite under various loadings. Figures 5 and 6 are the filtered images and skeletons, respectively. Figure 7 shows the specimen of three-point bending. Figure 8 shows the crack length for various loadings.
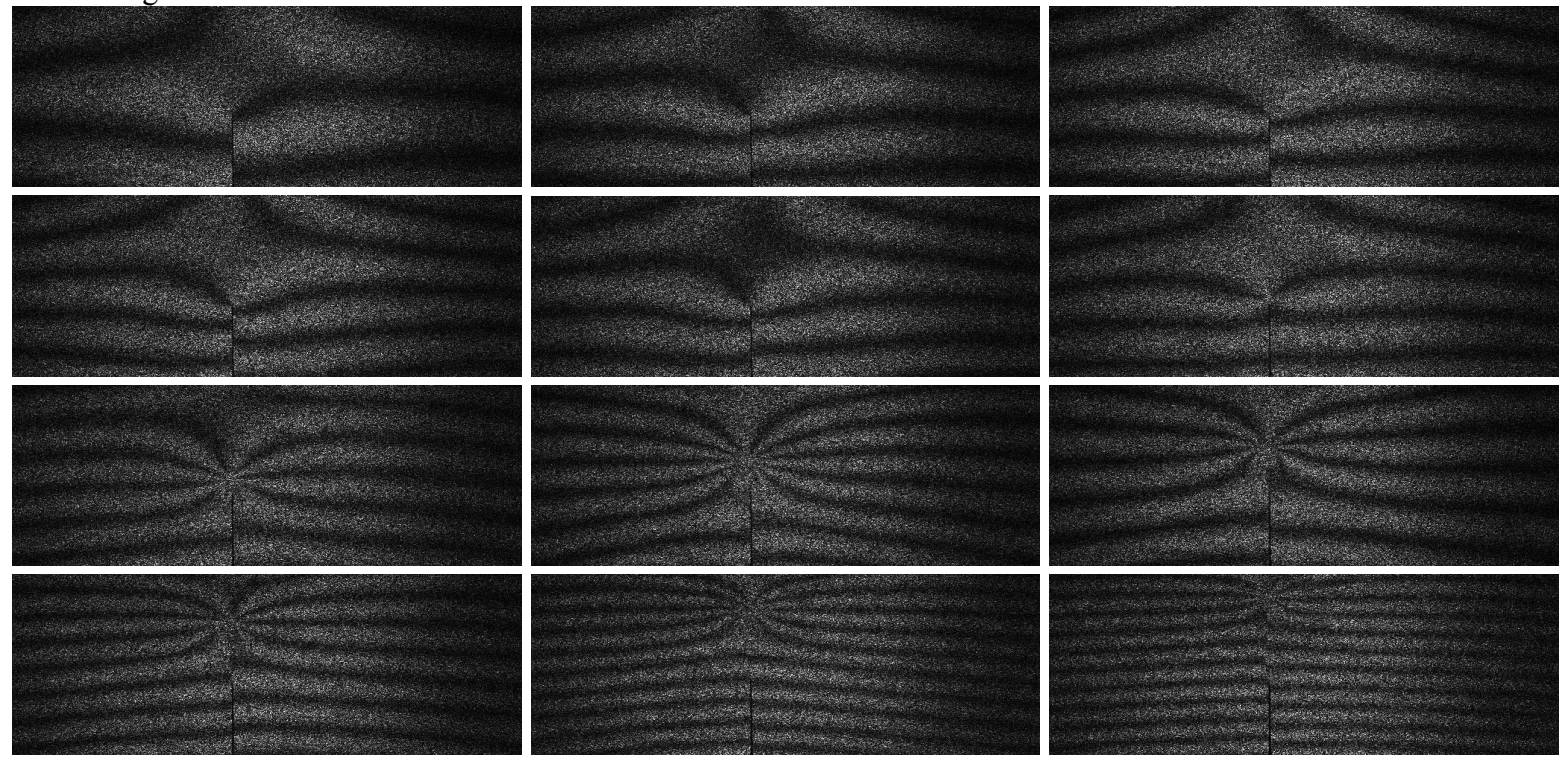

Figure 4. ESPI fringe images of fractured nuclear graphite under various loadings. 

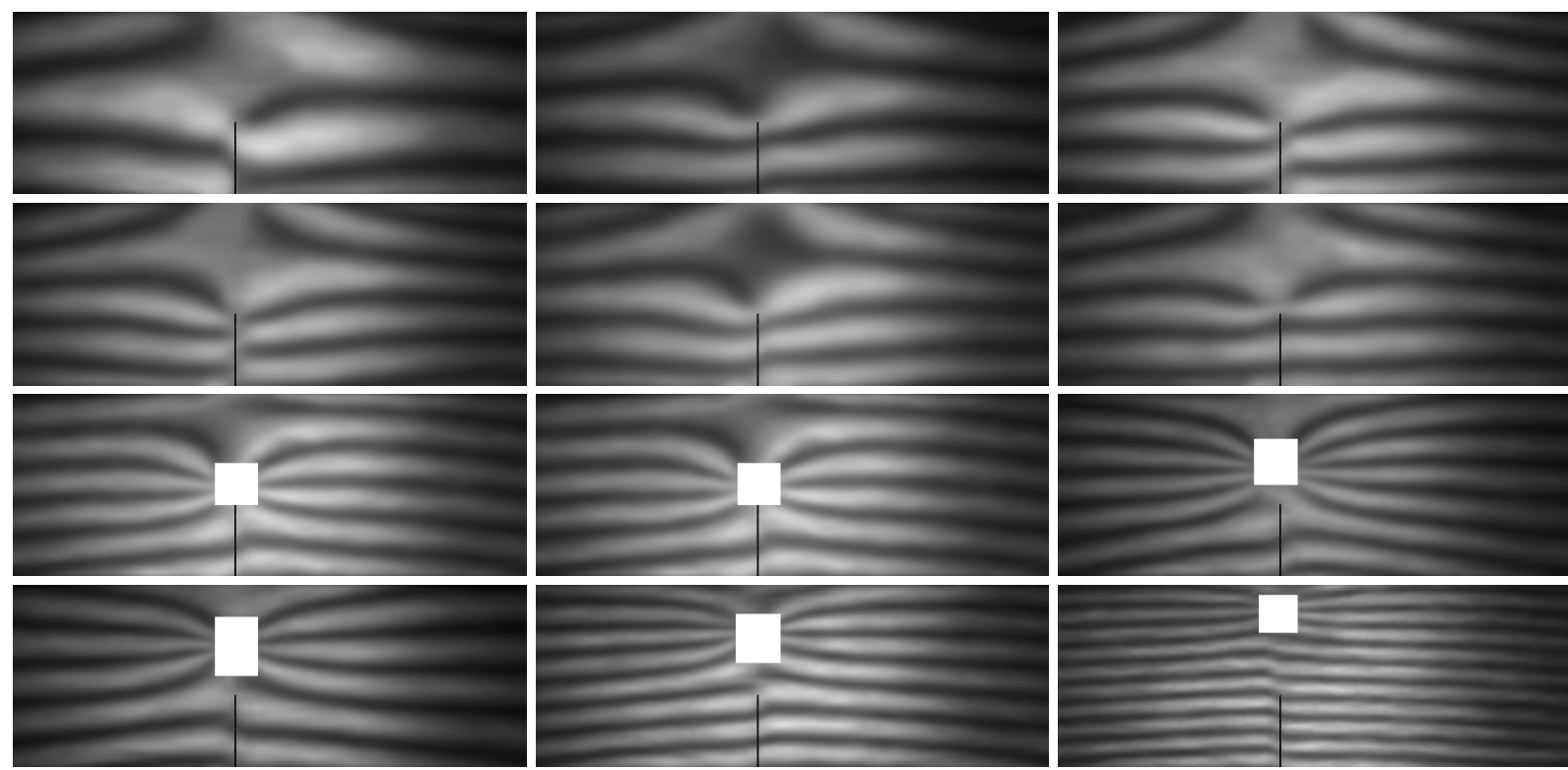

Figure 5. The corresponding filtered images of Figure 4.
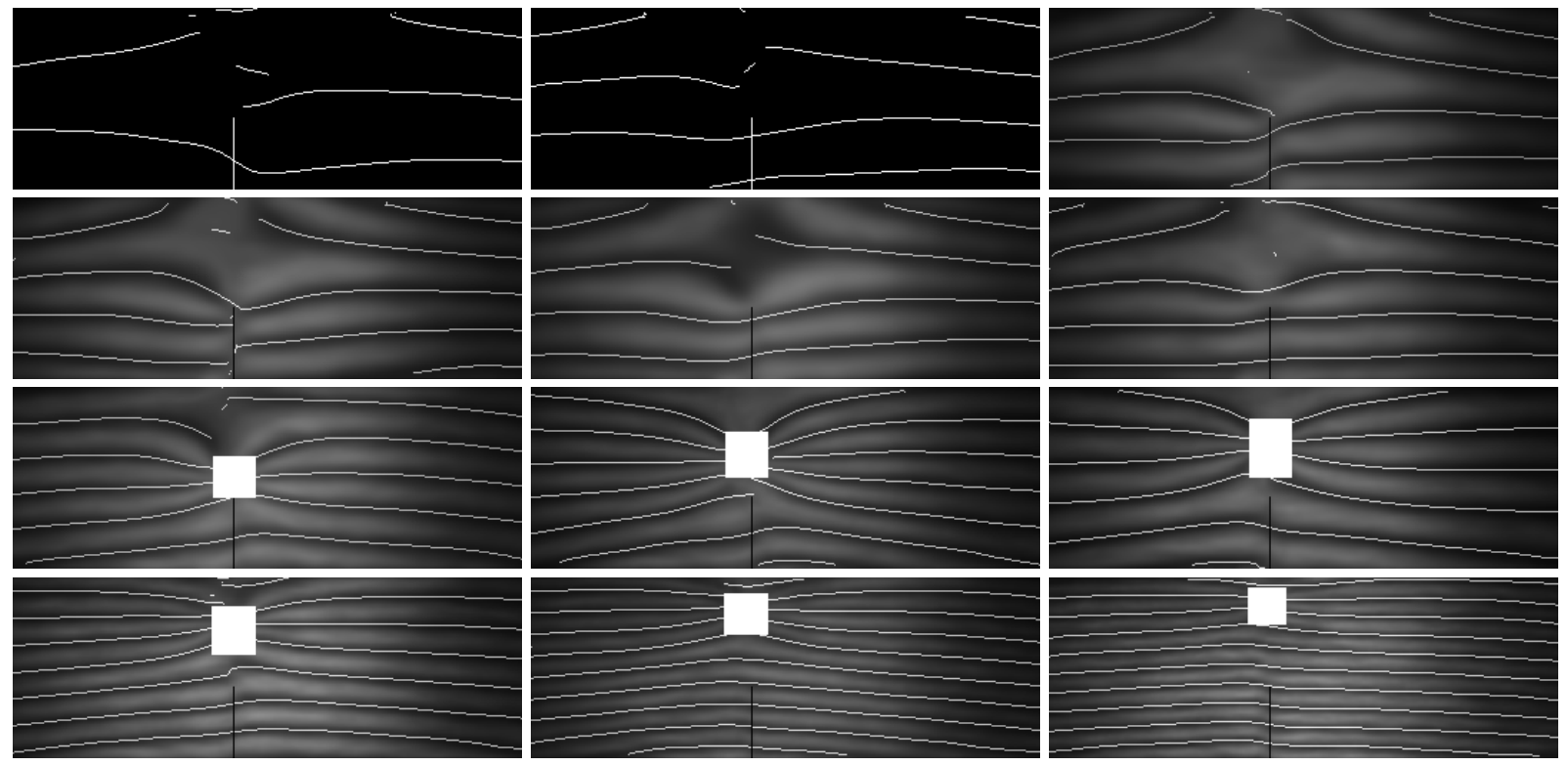

Figure 6. The corresponding skeletons of Figure 5.

Proc. of SPIE Vol. 9525 95252Y-7

Downloaded From: https://www.spiedigitallibrary.org/conference-proceedings-of-spie on 23 Jun 2019 Terms of Use: https://www.spiedigitallibrary.org/terms-of-use 


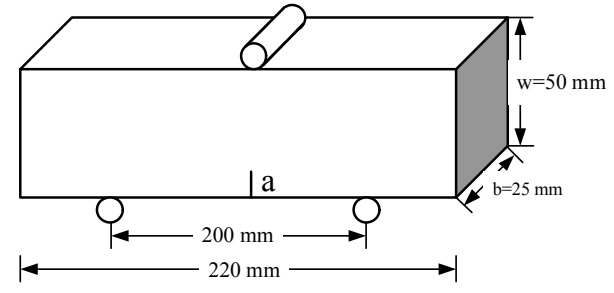

Figure 7. The specimen of three-point bending.

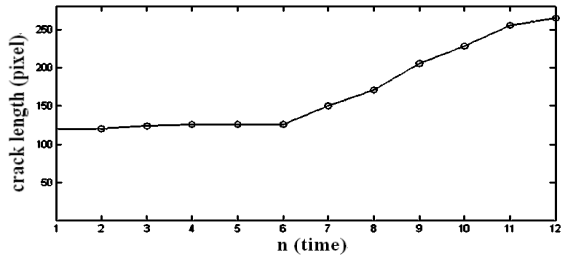

Figure 8 . The crack length for various loadings.

\section{CONCLUSION}

In this paper, we investigated the fracture expansion in nuclear graphite based on PDE image processing methods. We used the oriented bilateral filtering method and the second-order oriented partial differential equations filtering model (SOOPDE) to denoise speckle noise, then used the oriented gradient vector fields for to obtain skeletons. The full-field displacements of fractured nuclear graphite and the locations of the crack tip were lastly measured under various loading conditions.

\section{ACKNOWLEDGMENTS}

This work is supported by the National Science Foundation of China (NNSFC) (grant 61177007).

\section{REFERENCES}

[1] Aslan, M., "Toward the development of high-speed microscopic ESPI system for monitoring laser heating/drilling of alumina $\mathrm{Al}_{2} \mathrm{O}_{3}$ substrates," Ph. D. dissertation, Pennsylvania State University, UMI Number: 9966766 (2000).

[2] Tang, C., Han, L., Ren, H., Zhou, D., Chang, Y., Wang, X. and Cui, X., "Second-order oriented partial-differential equations for denoising in electronic-speckle-pattern interferometry fringes," Opt. Lett. 33(19), 2179-2181 (2008).

[3] Tomasi, C. and Manduchi, R., "Bilateral filtering for gray and color images," International Conference on Computer Vision, 839-846 (1998).

[4] Chang, H. and Chu, W., "Double bilateral filtering for image noise removal," World Congress on Computer Science and Information Engineering IEEE Computer Society 6, 451-455 (2009).

[5] Yu, Z. and Bajaj, C., "A segmentation-free approach for skeletonization of gray-scale images via anisotropic vector diffusion," Proceedings of the IEEE Conference on Computer Vision and Pattern Recognition 1, 415-420 (2004).

[6] Tang, C. Lu, W. Cai, Y. Han, L. and Wang, G., "Nearly preprocessing-free method for skeletonization of gray-scale electronic speckle pattern interferometry fringe patterns via partial differential equations," Opt. Lett. 33(2), 183-185 (2008).

[7] Tang, C., Ren, H. et al., "Oriented couple gradient vector fields for skeletonization of gray-scale optical fringe patterns with high density," Appl. Opt. 49(16),2979-2984 (2010).

[8] Wang, L., Leedham, G. and Cho, D. S. Y., "Minutiae feature analysis for infrared hand vein pattern biometrics," Pattern Recognition 41(3), 920-929 (2008). 